\title{
Evaluation of NOTCH3 Pro167Ser Variation in a Japanese Family with Cerebral Autosomal Dominant Arteriopathy with Subcortical Infarcts and Leukoencephalopathy
}

\author{
Toshiki Mizuno $^{a}$ Ikuko Mizuta $^{a}$ Hidekazu Tomimoto $^{b}$ \\ a Department of Neurology, Graduate School of Medical Science, Kyoto Prefectural \\ University of Medicine, Kyoto, and ${ }^{b}$ Department of Neurology, Mie University Graduate \\ School of Medicine, Tsu, Japan
}

Dear Editor,

Cerebral autosomal dominant arteriopathy with subcortical infarcts and leukoencephalopathy (CADASIL; OMIM\#125310) is one of the most common hereditary small-vessel diseases, caused by mutations of NOTCH3. In 2006, we reported three Japanese families, family 1, 2, and 3, clinically diagnosed with CADASIL in the article 'Small artery dementia in Japan: radiological differences between CADASIL, leukoaraiosis and Binswanger's disease' [1]. We identified Pro167Ser (P167S), Arg141Cys (R141C), and Arg75Pro (R75P) variation in each family. However, we did not clarify the pathogenic significance in that article. Here, we would like to address this issue.

It is well known that all CADASIL-causing mutations are localized in the epidermal growth factor-like repeat domain of $\mathrm{NOTCH} 3$, most of which result in the gain or loss of a cysteine residue in one of the repeats [2]. Interpretation of the cysteine-sparing mutations needs further analyses, including sequencing all exons, a segregation study, and pathological examinations [3]. Of the three variations reported by us [1], R141C is a typical pathogenic mutation of CADASIL. Although R75P is a cysteine-sparing mutation, its pathogenicity has been confirmed in several reports [4], including in a pathological skin biopsy examination we performed [5].

P167S variation was identified in family 1 . The mother suffered from cerebral infarction and her 2 daughters suffered from migraine. Their MRI showed characteristic findings of CADASIL. A genetic test was only performed for the mother who had suffered a stroke [1]. For genetic testing, four areas in the mutation hotspot region exon 3, 5' -half of exon 4, 3'-half of exon 4 , and exon 5 were analyzed by the single strand conformation polymorphism method for screening. The $5^{\prime}$-half of exon 4 was selected, and the subsequent sequencing identified P167S. Several years after our article had been published, we had the opportunity to conduct 
a genetic test of the patient's younger daughter. We performed genetic testing by sequencing exon 4. Surprisingly, not P167S but a typical pathogenic mutation, Arg182Cys (R182C), was identified in the daughter. Then, we sequenced all exons excluding exons 3-5 and identified another cysteine-sparing mutation, T1152M, in exon 21 only in the mother. Finally, we sequenced exons 3-5 and identified P167S and R182C in the mother. Because P167S and R182C exist in the $5^{\prime}$-half of exon 4 and $3^{\prime}$-half of exon 4, respectively, the reason for the missing R182C mutation in the previous genetic test of the mother may have been due to the sensitivity of the single strand conformation polymorphism method. We concluded that $\mathrm{R} 182 \mathrm{C}$ is the causative mutation in family 1 , and that P167S and T1152M are not pathogenic because there was no segregation with the affected mother and daughter.

\section{Disclosure Statement}

This research was supported by the construction and application of database for CADASIL, a hereditary small vessel disease from Japan Agency for Medical Research and development, AMED.

\section{References}

1 Tomimoto H, Ohtani R, Wakita H, Lin JX, Ihara M, Miki Y, Oshima F, Murata T, Ishibashi K, Suenaga T, Mizuno T: Small artery dementia in Japan: radiological differences between CADASIL, leukoaraiosis and Binswanger's disease. Dement Geriatr Cogn Disord 2006;21:162-169.

- Joutel A, Vahedi K, Corpechot C, Troesch A, Chabriat H, Vayssière C, Cruaud C, Maciazek J, Weissenbach J, Bousser MG, Bach JF, Tournier-Lasserve E: Strong clustering and stereotyped nature of NOTCH3 mutations in CADASIL patients. Lancet 1997;350:1511-1515.

3 GeneReviews ${ }^{\circledR}$ : CADASIL. http://www.ncbi.nlm.nih.gov/books/NBK1500/.

-4 Rutten JW, Haan J, Terwindt GM, van Duinen SG, Boon EM, Lesnik Oberstein SA: Interpretation of NOTCH3 mutations in the diagnosis of CADASIL. Expert Rev Mol Diagn 2014;14:593-603.

5 Mizuno T, Muranishi M, Torugun T, Tango H, Nagakane Y, Kudeken T, Kawase Y, Kawabe K, Oshima F, Yaoi T, Itoh K, Fushiki S, Nakagawa M: Two Japanese CADASIL families exhibiting NOTCH3 mutation R75P not involving cysteine residue. Intern Med 2008;47:2067-2072. 